ARTICLE

DOI: $10.1038 / s 41467-018-06976-7$

\title{
Rewriting the phase diagram of a diamagnetic liquid crystal by a magnetic field
}

\author{
Fatin Hajjaj', Takashi Kajitani (1) 1,2, Hiroyuki Ohsumi², Yoshikazu Tanaka², Kenichi Kato², Masaki Takata ${ }^{2,3}$, \\ Hideaki Kitazawa4 ${ }^{4}$ Taka-hisa Arima ${ }^{5,6}$, Takuzo Aida (D) ${ }^{6,7}$ \& Takanori Fukushima (D) ${ }^{1}$
}

Magnetic fields have been considered to only interact with organic materials non-destructively, leaving their fundamental structures unaffected, even when a strong magnetic field generated from a superconducting magnet is applied. Here we report an unprecedented observation that a liquid-crystalline mesophase of a diamagnetic molecular assembly with an orthorhombic or a cubic structure is formed selectively in the absence or presence of a strong magnetic field. The constituent molecule is a triphenylene derivative carrying six imidazolium bromide-terminated alkyl side chains and exhibits a cubic, orthorhombic, or hexagonal columnar mesophase when complexed with an appropriate amount of lanthanum(III) bromide. Thermal processing of the $\mathrm{La}^{3+}$-containing liquid-crystalline assembly in the presence of a 10-tesla magnetic field resulted in a phase diagram, in which the orthorhombic phase is completely replaced with the cubic phase. The discovery of this magneto-induced phaseselection offers an insight into the interactions between magnetic fields and organic material.

\footnotetext{
${ }^{1}$ Laboratory for Chemistry and Life Science, Institute of Innovative Research, Tokyo Institute of Technology, 4259 Nagatsuta, Midori-ku, Yokohama 2268503, Japan. ${ }^{2}$ RIKEN SPring-8 Center, 1-1-1 Kouto, Sayo, Hyogo 679-5148, Japan. ${ }^{3}$ Institute of Multidisciplinary Research for Advanced Materials, Tohoku University, 2-1-1 Katahira, Aoba-ku, Sendai 980-8577, Japan. ${ }^{4}$ National Institute for Materials Science, 1-2-1 Sengen, Tsukuba, Ibaraki 305-0047, Japan. ${ }^{5}$ Department of Advanced Materials Science, The University of Tokyo, 5-1-5 Kashiwanoha, Kashiwa, Chiba 277-8561, Japan. ${ }^{6}$ RIKEN Center for Emergent Matter Science (CEMS), 2-1 Hirosawa, Wako, Saitama 351-0198, Japan. ${ }^{7}$ Department of Chemistry and Biotechnology, School of Engineering, The University of Tokyo, 7-3-1 Hongo, Bunkyo-ku, Tokyo 113-8656, Japan. These authors contributed equally: Fatin Hajjaj, Takashi Kajitani. Correspondence and requests for materials should be addressed to T.A. (email: aida@macro.t.u-tokyo.ac.jp) or to T.F. (email: fukushima@res.titech.ac.jp)
} 
T he interactions between fields and matter represent not only a principal topic in fundamental science but also form the basis of functionality. Magnetic fields, for example, play a pivotal role in switching the physical properties of materials associated with $\operatorname{spin}^{1,2}$. In addition, magnetic fields can nondestructively orient mesoscopic entities ${ }^{3-5}$, even though they are intrinsically diamagnetic ${ }^{6-16}$. Examples include the magnetoinduced alignment of liquid-crystalline (LC) materials ${ }^{3,6-12}$, polymers 13,14 and organic/inorganic objects with a high aspect ratio ${ }^{4,5,13}$. The magneto-induced deformation of a spherical assembly of an amphiphile has also been reported ${ }^{17}$. However, the energy of magnetic fields has been considered too small to be able to influence molecular assembly processes under thermodynamic control. Accordingly, the resulting structures at the molecular level are generally independent of the presence or absence of a magnetic field, albeit only two exceptions have been reported for the occurrence of polymorphs during the solidification of organic molecules in magnetic fields ${ }^{18,19}$.

Here, we show an unprecedented phenomenon that a strong magnetic field can change the thermodynamically determined phase diagrams of diamagnetic liquid crystals. The liquid crystals consist of a discotic triphenylene core and six imidazolium bromide-terminated paraffinic side chains. Upon complexation with diamagnetic $\mathrm{LaBr}_{3}$, the resultant triphenylene-based ionic LC assemblies in the absence of a magnetic field exhibit a cubic, an orthorhombic or a hexagonal columnar mesophase, depending on the content of $\mathrm{LaBr}_{3}$ and temperature. When the LC composite with a certain content of $\mathrm{LaBr}_{3}$ was thermally processed under a strong magnetic field, the original phase diagram was dramatically changed, where the orthorhombic phase was fully replaced by a cubic phase. Through the investigation of the magneto-responsive phase behaviour of the diamagnetic liquid crystals, we demonstrate that the phase diagrams of diamagnetic molecular assemblies can be reprogrammed by applying a magnetic field.

\section{Results}

Design of the liquid crystals and initial observations of the magneto-responsive phase behaviour. Previously, we reported that paraffinic hexaalkoxytriphenylene derivatives carrying imidazolium pendants with $\mathrm{BF}_{4}^{-}$or $\mathrm{PF}_{6}{ }^{-}$counter anions (Supplementary Fig. 1) form LC assemblies that exhibit Ia $\overline{3} d$ cubic and $P 6 \mathrm{~mm}$ hexagonal columnar phases at lower and higher temperature, respectively ${ }^{20,21}$. Prior to these reports, only a few discotic molecules capable of forming I $a \overline{3} d$ cubic LC structures had been known ${ }^{22-24}$. We envisioned that such ionic LC assemblies $^{25-28}$ might become magneto-responsive if the counter anions were replaced with a paramagnetic metal ion. In particular, the hexagonal columnar assembly might be efficiently aligned in a magnetic field, although discotic columns are generally reluctant to align in external electric and magnetic fields ${ }^{9,10,29,30}$. With this expectation, we treated an imidazolium bromide-appended paraffinic triphenylene, $\operatorname{Im}_{\mathrm{TPBr}_{6}}$ (Fig. 1a), with paramagnetic $\mathrm{DyBr}_{3}$ at different molar ratios, $x=\mathrm{DyBr}_{3} /$ ${ }^{I m} \mathrm{TPBr}_{6}$, which furnished complex anion $\left[\mathrm{DyBr}_{6}\right]^{3-}$ quantitatively, to give $\left[\mathrm{DyBr}_{6}\right]^{3-}{ }_{x} \cdot\left(\mathrm{Br}^{-}\right)_{6-3 x}(x \leq 2.0)$ (Fig. 1a and see also Supplementary Note 3$)$. Depending on the molar ratio $(x)$ and the temperature, the obtained ${ }^{\operatorname{Im} T P}[\mathrm{Dy}]_{x}$ (Fig. 1a) showed a mesophase with an $I a \overline{3} d$ cubic (Cub, $0.0<x \leq 0.5$ ), a Pbcm orthorhombic (Ortho, $0.7 \leq x \leq 0.8$ ) or a $P 6 \mathrm{~mm}$ hexagonal columnar $\left(\mathrm{Col}_{\mathrm{h}}, x>1.0\right)$ structure, resulting in the phase diagram shown in
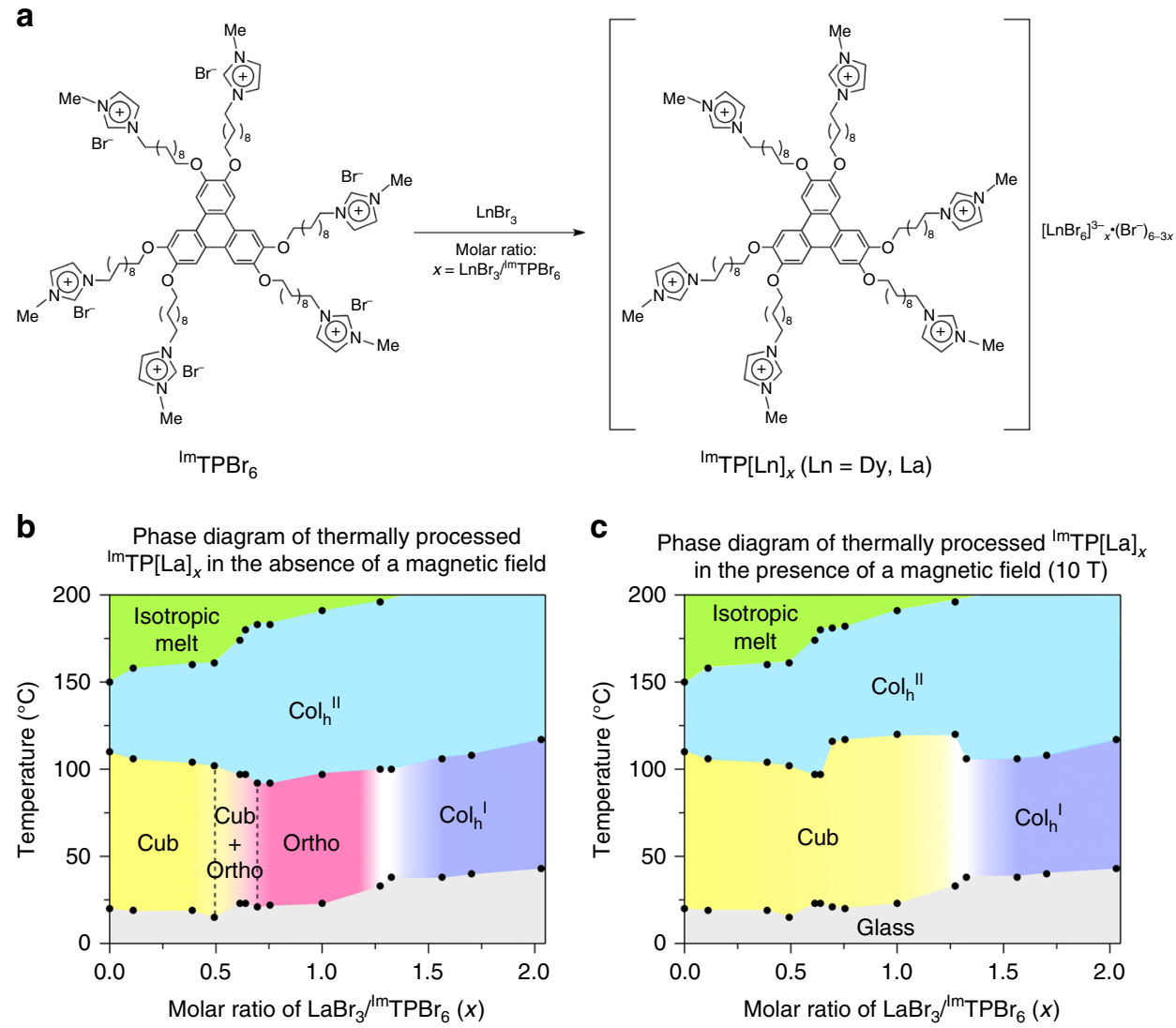

Fig. 1 Chemical structures of ${ }^{I m} \operatorname{TPBr}_{6}$ and ${ }^{I m} T P[L n]_{x}(L n=D y, L a)$, and phase diagrams of ${ }^{I m T P}[L a]_{x}$. a Preparation of composites ${ }^{I m T P}[L n]_{x}$. b, c Phase diagrams of $\left.\operatorname{ImTP}_{\mathrm{TLa}}\right]_{x}$ after thermal processing in the absence $(\mathbf{b})$ or presence (c) of a 10-T magnetic field. Cub cubic, Ortho orthorhombic, Col $\mathrm{h}_{\mathrm{h}}$ hexagonal columnar. The boundaries of the white regions could not be clearly determined 
Supplementary Fig. 2a (see also Supplementary Figs. 3-6). At 0.5 $<x<0.7,{ }^{I m} \mathrm{TP}[\mathrm{Dy}]_{x}$ displayed complex phase behaviour, in which either the Cub phase or the Ortho phase appeared stochastically or multiple domains of the two mesophases occurred simultaneously (Supplementary Fig. 7). During the magnetic alignment experiments, we serendipitously found an intriguing phenomenon: when ${ }^{\operatorname{Im}} \mathrm{TP}[\mathrm{Dy}]_{0.75}$ was heated once to $180^{\circ} \mathrm{C}$ and subsequently allowed to cool to $25^{\circ} \mathrm{C}$ (cooling rate $\leq 0.5^{\circ} \mathrm{C} / \mathrm{min}$ ) in a 10-tesla (T) magnet, the Ortho phase disappeared and a Cub phase emerged (Supplementary Figs. 2b, 8).

Initially, we thought that this unexpected phenomenon should arise from the paramagnetic nature of ${ }^{\operatorname{Im}} \mathrm{TP}[\mathrm{Dy}]_{x}$. However, we later noticed that even the diamagnetic analogue $\operatorname{Im}_{\mathrm{TP}}[\mathrm{La}]_{x}(x=$ $\mathrm{LaBr}_{3} / \mathrm{Im}_{\mathrm{TPBr}}$; Fig. 1a), which exhibits a more well-defined phase diagram than ${ }^{\mathrm{Im}} \mathrm{TP}[\mathrm{Dy}]_{x}$ (see Supplementary Discussion 1), displays a similar magneto-responsive phase behaviour, as shown in Fig. $1 b$, c. In brief, when ${ }^{I m} \mathrm{TP}[\mathrm{La}]_{x}$ was thermally processed in strong magnetic fields, an Ortho phase, which was originally observed in the phase diagram of $\operatorname{ImTP}[\mathrm{La}]_{x}$ at $0.7 \leq x<1.3$ (Fig. 1b), was completely replaced by a Cub phase (Fig. 1c).

Phase diagrams of ${ }^{\operatorname{Im}} \mathrm{TP}[\mathrm{La}]_{x}$ in the absence and presence of a magnetic field. Composites ${ }^{I m} \mathrm{TP}[\mathrm{La}]_{x}(0.0<x \leq 2.0)$ were prepared by mixing ${ }^{I m} \mathrm{TPBr}_{6}$ with appropriate amounts of $\mathrm{LaBr}_{3}$ in dehydrated methanol, followed by removal of the solvent under reduced pressure (see Supplementary Note 3). Based on analyses using synchrotron powder X-ray diffraction (PXRD), differential scanning calorimetry (DSC) and polarized optical microscopy $(\mathrm{POM})$, we established the phase diagram of ${ }^{\operatorname{Im} T P}[\mathrm{La}]_{x}$ as shown in Fig. 1b. At $x=2.0,{ }^{I m} \mathrm{TP}[\mathrm{La}]_{2.0}$ exhibited two different $\mathrm{Col}_{\mathrm{h}}$ phases at $43-117^{\circ} \mathrm{C}\left(\mathrm{Col}_{h} \mathrm{I}\right)$ and $117-222{ }^{\circ} \mathrm{C}\left(\mathrm{Col}_{h}{ }^{\mathrm{II}}\right)$ (Supplementary Fig. 9). Judging from the absence and presence of a broad diffraction peak arising from $\pi$-stacked triphenylene molecules, the $\mathrm{Col}_{h}^{\mathrm{I}}$ and $\mathrm{Col}_{h}{ }^{\mathrm{II}}$ phases feature disordered and ordered intracolumnar stacking, respectively (Fig. 2a, b). When the molar ratio was decreased $(0.7 \leq x<1.3)$, the $\mathrm{Col}_{h}{ }^{\mathrm{I}}$ phase disappeared and the Ortho phase emerged in the temperature region below the $\mathrm{Col}_{\mathrm{h}}$ II phase (Fig. $2 \mathrm{c}$ and Supplementary Figs. 10 and 11). At $0.0<x \leq 0.5,{ }^{I m} \mathrm{TP}[\mathrm{La}]_{x}$ exhibited the Cub phase in the temperature region below the $\mathrm{Col}_{h}{ }^{\mathrm{II}}$ phase (Fig. $2 \mathrm{~d}$ and Supplementary Fig. 12). The phase behaviour of ${ }^{I_{2}} \mathrm{TPBr}_{6}$, i.e. $x=$ 0.0 for ${ }^{\operatorname{Im}} \mathrm{TP}[\mathrm{La}]_{x}$ (Supplementary Fig. 16), was essentially identical to that of $\operatorname{ImTP}[\mathrm{La}]_{x}(0.0<x \leq 0.5)$. At $0.5<x<0.7$, either the Ortho or the Cub phase appeared stochastically. Most likely, the Ortho phase is not a kinetically formed metastable phase but rather a thermodynamically stable phase, since there was no change in the POM images of the Ortho phase of, e.g. ${ }^{\mathrm{Im}} \mathrm{TP}$ $[\mathrm{La}]_{0.75}$, even after it was allowed to stand at $90^{\circ} \mathrm{C}$ (just below the Ortho-to-Col ${ }_{h}{ }^{\mathrm{II}}$ phase-transition temperature) for $12 \mathrm{~h}$ or at $25^{\circ}$ $C$ for 2 years.

As shown in Fig. 1c, a magnetic field changed the phase diagram of diamagnetic $\operatorname{Im}^{\mathrm{TP}}[\mathrm{La}]_{x}(0.7 \leq x<1.3)$. A glass tube (diameter: $4 \mathrm{~mm}$ ) containing a bulk sample of ${ }^{\operatorname{Im}} \mathrm{TP}[\mathrm{La}]_{0.75}(5$ $\mathrm{mg}$ ), for example, was attached to the copper holder of a cryostat with Kapton tape (see Methods and Supplementary Fig. 17a). After the cryostat chamber was evacuated, the cryostat was placed at the centre of the bore (diameter: $10 \mathrm{~cm}$ ) of a 10-T superconducting magnet in the absence of a magnetic field. Subsequently, the sample was heated to $180^{\circ} \mathrm{C}$, which is just below the clearing point of ${ }^{\operatorname{Im}} \mathrm{TP}[\mathrm{La}]_{0.75}\left(182^{\circ} \mathrm{C}\right)$, and annealed at this temperature for $10 \mathrm{~min}$. Next, a magnetic field of $10 \mathrm{~T}$ was applied at the same temperature while the sample was cooled slowly to $25^{\circ} \mathrm{C}$ (cooling rate $\leq 0.5^{\circ} \mathrm{C} / \mathrm{min}$ ). The resulting sample at $27^{\circ} \mathrm{C}$ showed a PXRD pattern (Fig. 2e) that was substantially different from that observed for the original Ortho phase (Fig. 2c), but essentially identical to that of the Cub phase of $\operatorname{Im} \mathrm{TP}[\mathrm{La}]_{x}$ $(0.0<x \leq 0.5)$ at $27^{\circ} \mathrm{C}$ (Fig. $2 \mathrm{~d}$ ). The same result was obtained when $\operatorname{Im} \mathrm{TP}[\mathrm{La}]_{0.75}$ was subjected to sequential heating $\left(180^{\circ} \mathrm{C}\right)$ and cooling $\left(25^{\circ} \mathrm{C}\right)$ in the 10 -T magnetic field (Supplementary Fig. 13). When the thermally processed $\operatorname{Im} T P[L a]_{0.75}$ in the presence of a 10-T magnetic field was once heated to its isotropic melt temperature and subsequently cooled in the absence of a magnetic field, the original phase sequence of $\operatorname{Im} \mathrm{TP}[\mathrm{La}]_{0.75}$ (Supplementary Fig. 10) was observed again (Supplementary Fig. 14). Importantly, when a sample of $\operatorname{Im} \mathrm{TP}[\mathrm{La}]_{x}(0.0<x \leq 0.5$, Cub or $x>1.3, \mathrm{Col}_{h} \mathrm{I}$ ) was thermally processed in the presence of a 10-T magnetic field under conditions identical to those for $\operatorname{Im} \mathrm{TP}[\mathrm{La}]_{0.75}$, the original phase remained unchanged (Fig. 1b, c).

In situ observations of the phase change of ${ }^{\operatorname{Im}} \mathrm{TP}[\mathrm{La}]_{0.75}$ under a magnetic field. This magneto-assisted phase-selection was directly monitored by in situ POM in the 10-T magnet. A film sample of $\operatorname{Im} \mathrm{TP}[\mathrm{La}]_{0.75}$ (thickness: ca. $50 \mu \mathrm{m}$ ) on a glass substrate was placed at the centre of the bore (diameter: $10 \mathrm{~cm}$ ) in the $10-\mathrm{T}$ magnet in such a way that the substrate surface was oriented perpendicular to the magnetic flux (see Methods and Supplementary Fig. 17b). When the film was once heated to $\sim 180^{\circ} \mathrm{C}$, a birefringent texture of the $\mathrm{Col}_{h}{ }^{\mathrm{II}}$ phase of $\operatorname{Im}_{\mathrm{TP}}[\mathrm{La}]_{0.75}$ was observed (Fig. 3a, top). When a 10-T magnetic field was applied at the same temperature, the birefringent texture gradually disappeared, and a uniform orange-coloured POM image appeared (Fig. 3a). These observations indicate that the molecules are homogeneously aligned over the entire film. We presume that this change is due to a phase transition from the $\mathrm{Col}_{h}{ }^{\mathrm{II}}$ to a discotic nematic $\left(\mathrm{N}_{\mathrm{D}}\right)$ phase (see Supplementary Discussion 2), which is absent in the phase diagrams of $\operatorname{Im} \mathrm{TP}[\mathrm{La}]_{x}$ regardless of the presence or absence of a magnetic field during thermal processing (Fig. 1b, c). When the film sample was cooled in the 10-T magnet, a birefringent texture reappeared at $\sim 170{ }^{\circ} \mathrm{C}$, persisted to $\sim 160^{\circ} \mathrm{C}$, and then disappeared at $158^{\circ} \mathrm{C}$, giving rise to a dark POM image over the entire film down to $25^{\circ} \mathrm{C}$ (Fig. 3a). Obviously, the assembling structures of ${ }^{\mathrm{Im}} \mathrm{TP}[\mathrm{La}]_{0.75}$ in the $\mathrm{Col}_{h}{ }^{\mathrm{II}}$ and Ortho phases are optically anisotropic and display birefringent textures in POM, whereas that in the Cub phase is inherently optically isotropic to afford a dark POM image. Thus, the in situ POM observations in the presence of a 10-T magnetic field suggest that the Cub phase originates from the $\mathrm{Col}_{h}{ }^{\mathrm{II}}$ or the Ortho phase at $\sim 160^{\circ} \mathrm{C}$ (see Supplementary Discussion 3).

We also monitored the change in the diagnostic X-ray diffraction peaks of $\operatorname{Im}^{\mathrm{TP}}[\mathrm{La}]_{0.75}$ at $q=1.5-2.3 \mathrm{~nm}^{-1}$ during thermal processing in an applied magnetic field of $7 \mathrm{~T}$. For this in situ X-ray diffraction measurement, we designed a dedicated experimental setup by modifying an 8 -T magnet (bore diameter: $2.5 \mathrm{~cm}$ ) and a cryostat (see Methods and Supplementary Fig. 17c). At $27^{\circ} \mathrm{C}$, prior to application of the 7-T magnetic field, ImTP $[\mathrm{La}]_{0.75}$ showed broad peaks at $q=1.7-2.0 \mathrm{~nm}^{-1}$ (Fig. 3b), which were assigned to reflections $110,002,200$ and 111 of the $\mathrm{Pbcm}$ orthorhombic structure. Upon heating to $187^{\circ} \mathrm{C}$ in the absence of a magnetic field, these diffraction peaks disappeared, and a new broad peak at $q=1.96 \mathrm{~nm}^{-1}$ emerged due to the Ortho $\rightarrow \mathrm{Col}_{h}{ }^{\mathrm{II}}$ phase transition (Fig. 3c). The intensity of this diffraction peak became negligible when the sample was allowed to stand for 10 min at $187^{\circ} \mathrm{C}$ after the application of a 7-T magnetic field (Fig. 3d). This observation indicates that the material loses its two-dimensional (2D) hexagonal structural order, which is consistent with the formation of the $\mathrm{N}_{\mathrm{D}}$ phase observed by in situ POM. When the sample was subsequently cooled to $157^{\circ} \mathrm{C}$ in the presence of the magnetic field, a strong peak at $q=$ $1.96 \mathrm{~nm}^{-1}$ reappeared, together with a weak diffraction at $q=2.1$ $\mathrm{nm}^{-1}$ (Fig. 3e). Although the reciprocal $q$-spacing ratio of these 



Fig. 2 Powder X-ray diffraction (PXRD) patterns of bulk samples of ImTP[La $]_{x}$. a-f PXRD patterns and magnifications of the small-angle region (scattering vector $q=0.6-6 \mathrm{~nm}^{-1}$ ) of $\left.\operatorname{ImTP}_{\mathrm{m}} \mathrm{La}\right]_{2.0}$ at $27^{\circ} \mathrm{C}$ on first heating $(\mathbf{a}), \operatorname{Im}_{\mathrm{TP}}[\mathrm{La}]_{2.0}$ at $147^{\circ} \mathrm{C}$ on first heating $(\mathbf{b})$, ImTP $[\mathrm{La}]_{0.75}$ at $27^{\circ} \mathrm{C}$ on first heating $(\mathbf{c})$, $\operatorname{ImTP}[\mathrm{La}]_{0.5}$ at $27^{\circ} \mathrm{C}$ on first heating (d), ImTP $[\mathrm{La}]_{0.75}$ at $27^{\circ} \mathrm{C}$ on first heating after thermal processing in a $10-\mathrm{T}$ magnetic field (e) and ${ }^{\mathrm{ImTP}}[\mathrm{La}]_{0.75}$ at $27^{\circ}$ $\mathrm{C}$ after thermal processing in a 10-T magnetic field, followed by thermal processing in the absence of a magnetic field (f). Prior to the measurements, the samples in (a-d) were heated once to $180^{\circ} \mathrm{C}$ in the absence of a magnetic field. The samples in (e, $\mathbf{f}$ ) were heated once to $180^{\circ} \mathrm{C}$ in the absence of a magnetic field, and subsequently cooled to $25^{\circ} \mathrm{C}$ (cooling rate $\leq 0.5^{\circ} \mathrm{C} / \mathrm{min}$ ) in a 10-T magnetic field. Cub cubic, Ortho orthorhombic, Col hexagonal columnar, a.u. arbitrary unit. Indices of the reflections are shown in the magnifications of the PXRD patterns

two peaks $(\sqrt{6} 6 \sqrt{8})$ agrees well with that expected for reflections 211 and 220 of the $I a \overline{3} d$ cubic structure, their intensity ratio is different from that observed for the magnetically induced $\mathrm{Cub}$ phase of $\operatorname{Im}_{\mathrm{TP}}[\mathrm{La}]_{0.75}$ (Fig. 2e). The observed X-ray diffraction peaks can be explained by considering the coexistence of the $\mathrm{Col}_{h}{ }^{\mathrm{II}}$ and $\mathrm{Cub}$ phases at $157^{\circ} \mathrm{C}$ in the presence of the magnetic field. Importantly, upon further cooling to $27^{\circ} \mathrm{C}$, the intensity of the stronger peak decreased, whereas that of the weaker peak increased, resulting in an intensity ratio of 5.0 at $27^{\circ} \mathrm{C}$ (Fig. 3f), which is virtually identical to that observed for the magnetically induced Cub phase of ${ }^{\operatorname{Im}} \mathrm{TP}[\mathrm{La}]_{0.75}$ (4.8; Fig. 2e). Therefore, in the $7 \mathrm{~T}$ magnetic field, the Cub phase of ${ }^{\mathrm{Im}} \mathrm{TP}[\mathrm{La}]_{0.75}$ starts to emerge from the $\mathrm{Col}_{\mathrm{h}}{ }^{\mathrm{II}}$ phase at $\sim 160{ }^{\circ} \mathrm{C}$ and develops over the entire material at $27^{\circ} \mathrm{C}$. The diffraction peaks of the Cub phase persisted stably at $27^{\circ} \mathrm{C}$ even after the magnetic field intensity was decreased to $0.0 \mathrm{~T}$ (Fig. $3 \mathrm{~g}$ ). However, in a second heating and 
a
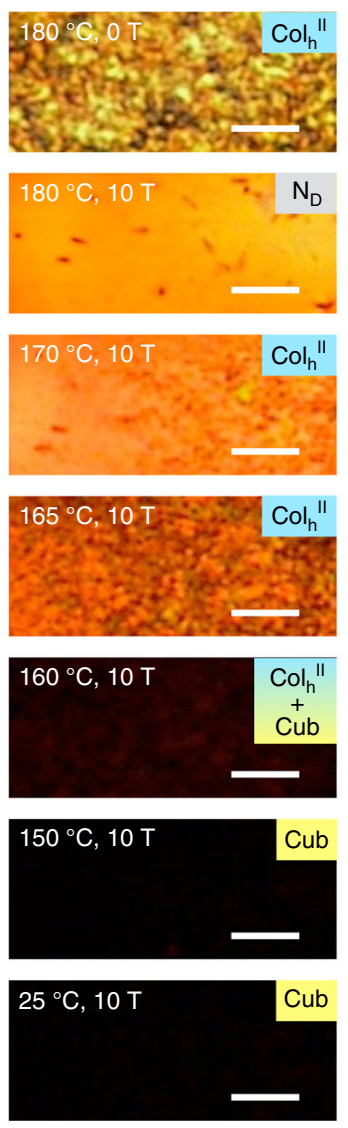

$\mathbf{k}$

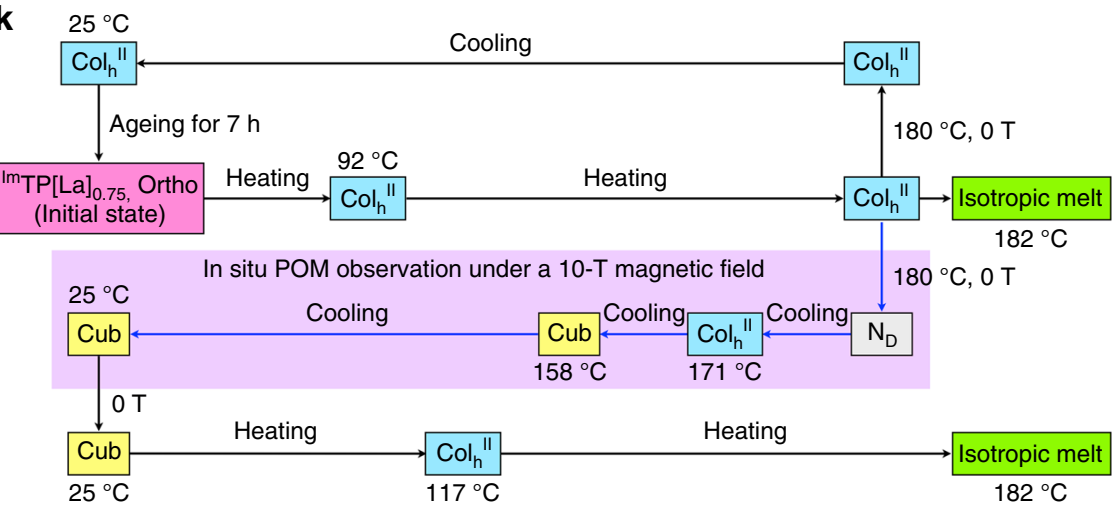

Fig. 3 In situ observations of the phase behaviour of ${ }^{\mathrm{m}} \mathrm{TP}[\mathrm{La}]_{0.75}$ under a strong magnetic field. a POM micrographs of a film of $\operatorname{lm} \mathrm{TP}[\mathrm{La}]_{0.75}$ on a glass substrate observed at $180^{\circ} \mathrm{C}$ (first heating; heating rate: $20^{\circ} \mathrm{C} / \mathrm{min}$ ) in the absence of a magnetic field, and observed at $180,170,165,160,150$ and $25^{\circ} \mathrm{C}$ upon cooling (cooling rate $\leq 0.5^{\circ} \mathrm{C} / \mathrm{min}$ ) in a 10-T magnetic field. Scale bars, $200 \mu \mathrm{m}$. b-j X-ray diffraction patterns of the small-angle region (scattering vector $q=1.5-2.3 \mathrm{~nm}^{-1}$ ) of a bulk sample of ImTP $[\text { La }]_{0.75}$ in a glass capillary (diameter: $2.5 \mathrm{~mm}$ ) measured at $27^{\circ} \mathrm{C}(\mathbf{b})$ and $187^{\circ} \mathrm{C}$ (c) upon first heating (heating rate: $20^{\circ} \mathrm{C} / \mathrm{min}$ ) in the absence of a magnetic field, measured at $187^{\circ} \mathrm{C}(\mathbf{d}), 157^{\circ} \mathrm{C}(\mathbf{e})$ and $27^{\circ} \mathrm{C}(\mathbf{f})$ upon first cooling from $187^{\circ} \mathrm{C}(\mathrm{cooling}$ rate: $0.5^{\circ} \mathrm{C} / \mathrm{min}$ ) in a $7-\mathrm{T}$ magnetic field, measured at $27^{\circ} \mathrm{C}(\mathbf{g})$ and $187^{\circ} \mathrm{C}(\mathbf{h})$ upon second heating (heating rate: $20^{\circ} \mathrm{C} / \mathrm{min}$ ) and measured at $27^{\circ} \mathrm{C}(\mathbf{i}, \mathbf{j}$ ) upon second cooling (cooling rate: $0.5^{\circ} \mathrm{C} / \mathrm{min}$ ) in the absence of a magnetic field. In (j), the measurement was conducted after ageing for $7 \mathrm{~h}$ at $27^{\circ} \mathrm{C}$. Ortho orthorhombic, Col hexagonal columnar, $\mathrm{N}_{\mathrm{D}}$ discotic nematic, Cub cubic, a.u. arbitrary unit. Indices of the reflections are shown at the top of the $\mathrm{X}$ ray diffraction peaks. $\mathbf{k}$ Schematic representation of the phase-transition behaviour of $\mathrm{ImTP}[\mathrm{La}]_{0.75}$ upon thermal processing in the absence or presence of a 10-T magnetic field. The phase-transition temperatures were determined based on POM observations in the absence or presence of the magnetic field

cooling cycle in the absence of a magnetic field, the system recovered the diffractions that were characteristic of the original Ortho phase (Fig. 3h-j). Meanwhile, we confirmed that, upon second heating in the presence of the 7-T magnetic field, the magnetically induced Cub phase of ${ }^{\mathrm{Im}} \mathrm{TP}[\mathrm{La}]_{0.75}$ is maintained up to at least $157^{\circ} \mathrm{C}$ (Supplementary Fig. 18), which is higher than the Cub-to-Col ${ }_{h}{ }^{\mathrm{I}}$ phase-transition temperature of $\operatorname{Im}_{\mathrm{TP}}[\mathrm{La}]_{x}(0.0$ $<x \leq 0.5)$ (Fig. 1b) as well as that of the magnetically induced Cub phase of ${ }^{\operatorname{Im}} \mathrm{TP}[\mathrm{La}]_{0.75}$, observed upon heating in the absence of a magnetic field. The fact that the temperature range of the Cub phase expands in the presence of the magnetic field suggests this phase is substantially stabilized under the conditions employed. 


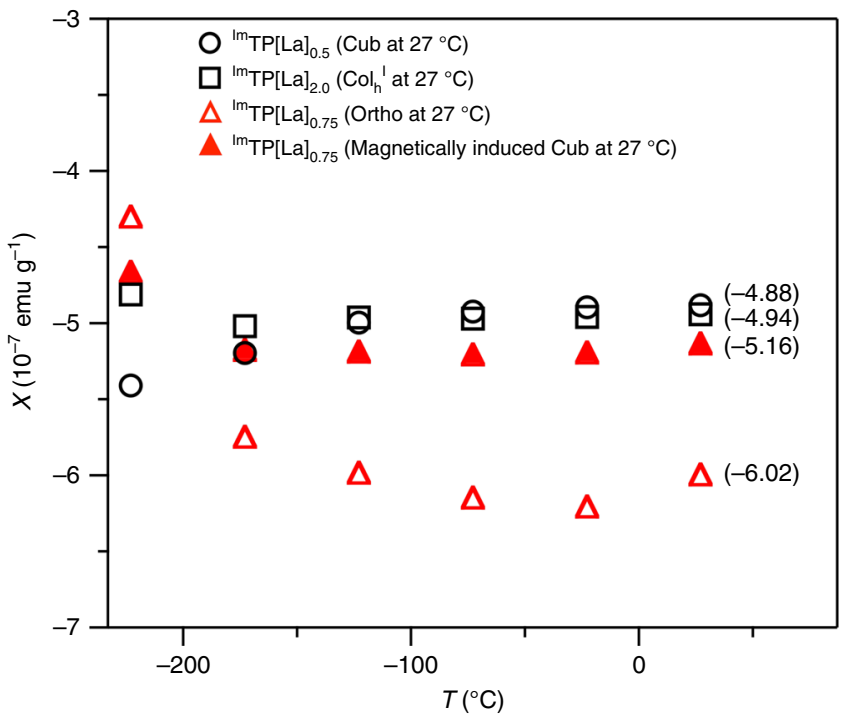

Fig. 4 SQUID measurements of ${ }^{I m} T P[L a]_{x} \cdot \chi-T$ curves for ${ }^{I m} T P[L a]_{x}$ after thermal processing in the absence of a magnetic field ( $x=0.5$ : open circle, $x=2.0$ : open square and $x=0.75$ : open triangle) and for ${ }^{I m T P}[L a]_{0.75}$ after thermal processing in a 10-T magnetic field (filled triangle). Values in parentheses represent the $\chi$ values $\left(10^{-7} \mathrm{emu} / \mathrm{g}\right)$ of the samples at $27^{\circ} \mathrm{C}$

Magneto-responsive phase behaviour of ${ }^{\operatorname{Im}} \mathrm{TP}[\mathrm{La}]_{0.75}$. Based on an experiment using a bulk sample of ${ }^{I m} \mathrm{TP}[\mathrm{La}]_{0.75}$ in a glass capillary (diameter: $0.7 \mathrm{~mm}$ ), the magnetically induced Cub phase of $\operatorname{Im}_{\mathrm{TP}}[\mathrm{La}]_{0.75}$ did not spontaneously transform back into the Ortho phase even after standing at $25^{\circ} \mathrm{C}$ for more than 3 months outside the magnet, while it changed after heating to $180^{\circ} \mathrm{C}$ and subsequent cooling to $25^{\circ} \mathrm{C}$ in the absence of a magnetic field, resulting in a PXRD pattern (Fig. 2f), which is identical to that of the original Ortho phase of $\operatorname{Im}_{\mathrm{TP}}[\mathrm{La}]_{0.75}$ (Fig. $2 \mathrm{c}$ ). These results obtained by in situ POM and XRD experiments using a film and a bulk sample of ${ }^{\operatorname{Im}} \mathrm{TP}[\mathrm{La}]_{0.75}$, respectively, clearly indicate that the structural information of the LC material, memorized under the application of a magnetic field, can be initialized by thermal processing without a magnetic field.

With the above results in mind, we can now provide a full picture of the magneto-responsive phase behaviour of ImTP $[\mathrm{La}]_{0.75}$, as illustrated in Fig. $3 \mathrm{k}$. Notably, the magneto-assisted phase-selection depends critically on the strength of the applied magnetic field as well as on the annealing temperature. For instance, upon thermal processing with a 5-T magnetic field, the Cub phase of $\operatorname{Im}_{\mathrm{TP}}[\mathrm{La}]_{0.75}$ was stochastically selected over the Ortho phase and, in some cases, an LC assembly composed of a mixture of domains of the Ortho and Cub phases was obtained (Supplementary Fig. 15a). In a magnetic field stronger than $5 \mathrm{~T}$, only the Cub phase emerged. Accordingly, the magneto-assisted phase-selection of $\operatorname{Im}_{\mathrm{TP}}[\mathrm{La}]_{0.75}$ might require magnetic fields stronger than $5 \mathrm{~T}$. Meanwhile, when ${ }^{\operatorname{Im}} \mathrm{TP}[\mathrm{La}]_{0.75}$ was annealed for $10 \mathrm{~min}$ at $150^{\circ} \mathrm{C}$ in a $10-\mathrm{T}$ magnetic field, a mixture of domains of the Ortho and Cub phases formed (Supplementary Fig. 15b). Upon annealing for $10 \mathrm{~min}$ at a lower temperature (e.g. $90^{\circ} \mathrm{C}$ ), the original Ortho phase of ${ }^{\operatorname{Im}} \mathrm{TP}[\mathrm{La}]_{0.75}$ was maintained even after thermal processing in a $10-\mathrm{T}$ magnetic field (Supplementary Fig. 15c).

Superconducting quantum interference device (SQUID) measurements of $\operatorname{Im}_{\mathrm{TP}}[\mathrm{La}]_{x}$. To gain insight into this magnetoassisted phase-selection, we evaluated the effect of a magnetic field on the free energy $\left(E_{\mathrm{mag}}\right)$ of ${ }^{\operatorname{Im}} \mathrm{TP}[\mathrm{La}]_{x}$ using the equation $E_{\text {mag }}=-\chi B^{2} / 2 \mu_{0}$, where $\chi$ is the mass magnetic susceptibility, $B$ is the magnetic flux density and the physical constant $\mu_{0}$ is the magnetic permeability of vacuum ${ }^{15,31}$. Using a SQUID (see Methods), we measured $\chi$ values at various temperatures for ${ }^{I m} \mathrm{TP}$ $[\mathrm{La}]_{x}(x=0.5,0.75$ and 2.0$)$ after thermal processing in the absence of a magnetic field, as well as that of ${ }^{\operatorname{Im}} \mathrm{TP}[\mathrm{La}]_{0.75}$ after thermal processing in a 10-T magnetic field (Fig. 4). We confirmed that no macroscopic alignment of the anisotropic Ortho phase occurs at the measurement temperature range even under the application of a 5-T magnetic field, and thus the SQUID profiles reflect the intrinsic magnetic properties of the materials. Due to the diamagnetic nature of ${ }^{\operatorname{Im}} \mathrm{TP}[\mathrm{La}]_{x}$, the $\chi$ value for each sample was negative at all temperatures examined. The diamagnetic signals, $|\chi|$, of ${ }^{\operatorname{Im}} \mathrm{TP}[\mathrm{La}]_{0.5}$ and $\operatorname{Im}_{\mathrm{TP}}[\mathrm{La}]_{2.0}$, which adopt $\mathrm{Cub}$ and $\mathrm{Col}_{\mathrm{h}}{ }^{\mathrm{I}}$ structures at $27^{\circ} \mathrm{C}$, respectively, were comparable to each other. The $|\chi|$ values of $\operatorname{Im}^{\mathrm{TP}}[\mathrm{La}]_{0.75}$, which originally adopts the Ortho structure at $27^{\circ} \mathrm{C}$, were slightly greater than those of ${ }^{\operatorname{Im}} \mathrm{TP}[\mathrm{La}]_{0.5}$ and ${ }^{\operatorname{Im}} \mathrm{TP}[\mathrm{La}]_{2.0}$. The $\chi$ values of ${ }^{\operatorname{Im}} \mathrm{TP}[\mathrm{La}]_{0.5}$, $\operatorname{Im}_{\mathrm{TP}}[\mathrm{La}]_{2.0}$ and ${ }^{\operatorname{Im}} \mathrm{TP}[\mathrm{La}]_{0.75}$ at $27^{\circ} \mathrm{C}$ were determined to be $-4.88 \times 10^{-7},-4.94 \times 10^{-7}$ and $-6.02 \times 10^{-7} \mathrm{emu} / \mathrm{g}$, respectively. Since the $B^{2} / 2 \mu_{0}$ values are identical under the measurement conditions, the Ortho phase of ${ }^{\operatorname{Im}} \mathrm{TP}[\mathrm{La}]_{0.75}$ should be more destabilized than the other LC phases in terms of $E_{\text {mag. }}$. Remarkably, when ${ }^{\operatorname{Im}} \mathrm{TP}[\mathrm{La}]_{0.75}$ was heated to $180^{\circ} \mathrm{C}$ and subsequently cooled to $25^{\circ} \mathrm{C}$ in the presence of a 10-T magnetic field, the resulting material showed a $\chi-T$ curve similar to that observed for the Cub phase of ${ }^{\operatorname{Im}} \mathrm{TP}[\mathrm{La}]_{0.5}$ (Fig. 4). Furthermore, the $|\chi|$ value of ${ }^{\operatorname{Im}} \mathrm{TP}[\mathrm{La}]_{0.75}$ at $27^{\circ} \mathrm{C}$ was decreased from $6.02 \times 10^{-7}$ to $5.16 \times 10^{-7} \mathrm{emu} / \mathrm{g}$ after thermal processing in the presence of a 10 -T magnetic field. The change in the $\chi-T$ curve for ${ }^{\operatorname{Im}} \mathrm{TP}[\mathrm{La}]_{0.75}$ before and after thermal processing in the magnetic field strongly supports the occurrence of magneto-assisted phase-selection, where the initial Ortho phase is reprogrammed into the Cub phase (Fig. 3k).

\section{Discussion}

Based on an analogy to conventional thermotropic and lyotropic liquid crystals that exhibit a phase transition in response to thermal stimuli and the presence of solvents, respectively, the unprecedented phase behaviour of the present LC material can be referred to as 'magnetotropic'. The discovery that a magnetic field can change the phase behaviour of a diamagnetic molecular assembly should not only impact various scientific areas such as chemistry, condensed matter physics and materials science, but also substantially expand the understanding of the interplay between soft matter and magnetic fields, which could ultimately provide a concept for the design of organic materials.

\section{Methods}

Thermal processing of ${ }^{I m} \mathrm{TP}[\mathrm{Ln}]_{x}$ in the presence of a 10-T magnetic field. A glass tube (diameter: $4 \mathrm{~mm}$ ), containing a bulk sample of $\operatorname{Im}_{\mathrm{TP}}[\mathrm{Ln}]_{x}(5 \mathrm{mg})$, was attached to the copper sample holder of a cryostat chamber (ST-300; JANIS Research Co., Inc.) with Kapton tape (Supplementary Fig. 17a). The cryostat chamber was subsequently evacuated by a turbo-molecular pump to maintain a pressure of $\sim 10^{-4} \mathrm{mbar}$, before being placed at the centre of the bore (diameter: 10 $\mathrm{cm}$ ) of a 10-T superconducting magnet (JMTD-10T100RK; JASTEC, Inc.) in such a way that the long axis of the glass tube containing the sample was oriented paralle to the magnetic flux. The temperature of the sample holder was controlled with an external temperature-control unit (1900-7; Scientific Instruments, Inc.). Unless otherwise stated, the sample was heated once to $180^{\circ} \mathrm{C}$ in the absence of a magnetic field, before being cooled to $25^{\circ} \mathrm{C}$ (cooling rate $\leq 0.5^{\circ} \mathrm{C} / \mathrm{min}$ ) in the presence of a 10-T magnetic field. After thermal processing, the sample was collected and stored under argon in a sealed vial at $25^{\circ} \mathrm{C}$.

Synchrotron radiation powder X-ray diffraction (PXRD) experiments of bulk samples of ${ }^{I m} \mathbf{T P B r}_{6}$ and ${ }^{I m} \mathbf{T P}[\mathbf{L n}]_{\mathbf{x}}(\mathbf{L n}=$ Dy or $\mathbf{L a})$. Variable-temperature onedimensional (1D) X-ray diffraction patterns were measured using beamline 44B2 in SPring-8 (Hyogo, Japan) equipped with an imaging-plate area detector ${ }^{32}$. The wavelength (1.08 $\AA$ ) of incident X-rays was calibrated using cerium oxide (standard reference material $674 \mathrm{~b}$ ). The sample-to-detector distance was $286.5(1) \mathrm{mm}$. Unless 
otherwise stated, bulk samples in a glass capillary (diameter: $0.7 \mathrm{~mm}$ ) were measured while spinning at a rate of $60 \mathrm{rpm}$.

\section{Structural characterization of the Ortho phases of ImTP[Dy $]_{0.75}$ and ImTP} [La $]_{\mathbf{0 . 7 5}}$. A shear-oriented film sample of ${ }^{\operatorname{Im}} \mathrm{TP}[\mathrm{Dy}]_{0.75}$ or ${ }^{\mathrm{Im}} \mathrm{TP}[\mathrm{La}]_{0.75}$ was prepared by applying a shear stress to a bulk material of ${ }^{\operatorname{Im}} \mathrm{TP}[\mathrm{Dy}]_{0.75}$ or ${ }^{\operatorname{Im}} \mathrm{TP}[\mathrm{La}]_{0.75}$ at $150{ }^{\circ} \mathrm{C}$ on a sapphire substrate, and the resulting film was slowly cooled to $25^{\circ} \mathrm{C}$ and aged at $25^{\circ} \mathrm{C}$ for several days. A $2 \mathrm{D}$ X-ray diffraction image of the film (Supplementary Fig. 5 for ${ }^{\operatorname{Im}} \mathrm{TP}[\mathrm{Dy}]_{0.75}$ or Supplementary Fig. 11 for $\operatorname{Im}_{\mathrm{TP}}[\mathrm{La}]_{0.75}$ ) was obtained using beamline 45XU in SPring-8 (Hyogo, Japan) equipped with an $\mathrm{R}$-AXIS IV++ (Rigaku) imaging-plate area detector. The scattering vector, $q=$ $4 \pi \sin \theta / \lambda$, and the position of the incident X-ray beam on the detector were calibrated using several orders of layer reflections from silver behenate $(d=58.380 \AA)$, where $2 \theta$ and $\lambda$ refer to the scattering angle and wavelength of the X-ray beam (1.0 $\AA$ ), respectively. The sample-to-detector distance was $0.5 \mathrm{~m}$. The cell parameters were refined using CellCalc ver. 2.10 software $^{33}$.

In situ polarized optical microscopic (POM) observation in the presence of a 10-T magnetic field. To visualize the phase-transition events of ${ }^{\mathrm{Im}} \mathrm{TP}[\mathrm{La}]_{0.75}$ inside a 10-T magnet (JMTD-10T100RK; JASTEC, Inc.), we designed a dedicated heater unit (Supplementary Fig. 17b, left) consisting of a heater block, polariser and borescope (TechnoPack X; Karl Storz Co.). A film of $\operatorname{Im}_{\mathrm{TP}}[\mathrm{La}]_{0.75}$ on a $100 \mu \mathrm{m}$ thick glass substrate was attached to the heater block (Supplementary Fig. 17b, left). The heater was then placed inside the bore of the magnet (Supplementary Fig. 17b, right) in such a way that the surface of the substrate was oriented perpendicular to the magnetic flux, while the film sample was located at the centre of the bore. Due to the limitations of the experimental setup used, the exact temperature could not be ascertained.

In situ X-ray diffraction measurement in the presence of a 7-T magnetic field. In situ variable-temperature X-ray diffraction experiments in the presence of a magnetic field were carried out using beamline 19LXU in SPring-8 (Hyogo, Japan) ${ }^{34}$. An Oxford cryostat (Spectrostat-HT500V) and a rod-type sample holder were specifically modified and prepared, respectively, so as to adapt to the high-temperature measurements in a superconducting magnet. A bulk sample of $\operatorname{Im}_{\mathrm{TP}}[\mathrm{La}]_{0.75}$ in a glass capillary (diameter: $2.5 \mathrm{~mm}$ ) was placed inside a copper tube (Supplementary Fig. 17c, left). The tube was then attached to the rod-type sample holder (Supplementary Fig. $17 \mathrm{c}$, centre) and inserted into the modified cryostat (Supplementary Fig. 17c, right). The cryostat containing the sample was subsequently placed inside a $25-\mathrm{mm}$ variable-temperature insert (VTI) sample space of an 8-T superconducting magnet (PN5090; Oxford Instruments), containing windows of multilayer films of beryllium and Kapton. The superconducting magnet was then mounted on a HUBER crystallography diffractometer equipped with an avalanche photodiode (APD) detector (Supplementary Fig. $17 \mathrm{c}$, right). The VTI sample space ( 1 mbar) and the cryostat chamber were evacuated $\left(\sim 10^{-5} \mathrm{mbar}\right)$, and the temperature of the sample was controlled by an external temperature-control unit (Model 335 Cryogenic Temperature Controller; Lake Shore Cryotronics, Inc.). Due to the limitations of the experimental setup used, the exact temperature could not be ascertained. The sample was exposed to the X-ray beam (Si double-crystal monochromated X-rays: $\lambda=1.0 \AA$ ) through the windows of the magnet, VTI sample space and cryostat. The X-ray diffraction patterns were collected using the APD detector (sample-to-detector distance: $1.75 \mathrm{~m}$ )

Superconducting quantum interference device (SQUID) measurements. The mass magnetic susceptibility $\chi(T)$ of $\operatorname{Im} \mathrm{TP}[\mathrm{La}]_{x}$ as a function of temperature $T$ was measured using a Quantum Design MPMS-5S SQUID magnetometer with a maximum field of $5 \mathrm{~T}$. The $\chi-T$ curve of each ${ }^{\mathrm{Im}} \mathrm{TP}[\mathrm{La}]_{x}$ sample was measured upon cooling (cooling rate: $0.5^{\circ} \mathrm{C} / \mathrm{min}$ ) from 27 to $-223^{\circ} \mathrm{C}$ in the presence of a magnetic field $(B=0.5 \mathrm{~T})$. Prior to each measurement, bulk samples $(5-13 \mathrm{mg})$ of $\operatorname{Im} \mathrm{TP}[\mathrm{La}]_{x}$ were thermally processed at $180^{\circ} \mathrm{C}$ either in the presence or absence of a 10-T magnetic field. Each sample was packed in a glass capillary (ca. $8 \mathrm{~mm}$ in height, ca. $3 \mathrm{~mm}$ in outside diameter and ca. $30 \mathrm{mg}$ in weight) using a non-metallic toothpick to avoid magnetic contamination from the outside. The glass capillary containing the sample was sandwiched between double straws made of Kapton film (ca. $180 \mathrm{~mm}$ in length and $0.1 \mathrm{~mm}$ in thickness) and fixed in the centre of the straws. For all measurements, the Reciprocating Sample Option (RSO) with 4-cm sample movement and $1 \mathrm{~Hz}$ repetition frequency were used to achieve the high sensitivity of $\sim 10^{-8} \mathrm{emu}$. The magnetization of the glass capillary alone was measured first, then the magnetization of the sample was measured in the glass capillary, and the magnetization of only the sample was obtained from the difference between them.

\section{Data availability}

All relevant data are included in full within this paper and in the Supplementary Information.
Received: 11 April 2018 Accepted: 5 October 2018

Published online: 25 October 2018

\section{References}

1. Uji, S. et al. Magnetic-field-induced superconductivity in a two-dimensional organic conductor. Nature 410, 908-910 (2001).

2. Kimura, T. et al. Magnetic control of ferroelectric polarization. Nature 426, 55-58 (2003)

3. Binnemans, K. et al. Rare-earth-containing magnetic liquid crystals. J. Am. Chem. Soc. 122, 4335-4344 (2000).

4. Reches, M. \& Gazit, E. Controlled patterning of aligned self-assembled peptide nanotubes. Nat. Nanotechnol. 1, 195-200 (2006)

5. Beardslee, J. A., Sadtler, B. \& Lewis, N. S. Magnetic field alignment of randomly oriented, high aspect ratio silicon microwires into vertically oriented arrays. ACS Nano 6, 10303-10310 (2012).

6. Tolbert, S. H., Firouzi, A., Stucky, G. D. \& Chmelka, B. F. Magnetic field alignment of ordered silicate-surfactant composites and mesoporous silica. Science 278, 264-268 (1997)

7. Boamfa, M. I., Lazarenko, S. V., Vermolen, E. C. M., Kirilyuk, A. \& Rasing, T. Magnetic field alignment of liquid crystals for fast display applications. $A d v$. Mater. 17, 610-614 (2005).

8. Shklyarevskiy, I. O. et al. High anisotropy of the field-effect transistor mobility in magnetically aligned discotic liquid-crystalline semiconductors. J. Am. Chem. Soc. 127, 16233-16237 (2005).

9. Kim, H.-S. et al. Uniaxially oriented, highly ordered, large area columnar superstructures of discotic supramolecules using magnetic field and surface interactions. Adv. Mater. 20, 1105-1109 (2008).

10. Bisoyi, H. K. \& Li, Q. Intelligent Stimuli-responsive Materials: From Welldefined Nanostructures to Applications Ch. 3 (Wiley-VCH, Hoboken 2013).

11. Salili, S. M. et al. Magnetically tunable selective reflection of light by heliconical cholesterics. Phys. Rev. E 94, 042705 (2016).

12. Bisoyi, H. K., Bunning, T. J. \& Li, Q. Stimuli-driven control of the helical axis of self-organized soft helical superstructures. Adv. Mater. 30, 1706512 (2018).

13. Kimura, T. et al. Polymer composites of carbon nanotubes aligned by a magnetic field. Adv. Mater. 14, 1380-1383 (2002)

14. Majewski, P. W., Gopinadhan, M., Jang, W. S., Lutkenhaus, J. L. \& Osuji, C. O. Anisotropic ionic conductivity in block copolymer membranes by magnetic field alignment. J. Am. Chem. Soc. 132, 17516-17522 (2010).

15. Micali, N. et al. Selection of supramolecular chirality by application of rotational and magnetic forces. Nat. Chem. 4, 201-207 (2012).

16. Liu, M. et al. An anisotropic hydrogel with electrostatic repulsion between cofacially aligned nanosheets. Nature 517, 68-72 (2015).

17. Shklyarevskiy, I. O. et al. Magnetic deformation of self-assembled sexithiophene spherical nanocapsules. J. Am. Chem. Soc. 127, 1112-1113 (2005).

18. Honjo, S., Yokota, M., Doki, N. \& Shimizu, K. Magnetic field influence on the crystal structure of $2,2^{\prime}: 6^{\prime}, 2^{\prime \prime}$-terpyridine. Kagaku Kogaku Ronbun 34, 383-387 (2008).

19. Potticary, J. et al. An unforeseen polymorph of coronene by the application of magnetic fields during crystal growth. Nat. Commun. 7, 11555 (2016).

20. Motoyanagi, J., Fukushima, T. \& Aida, T. Discotic liquid crystals stabilized by interionic interactions: imidazolium ion-anchored paraffinic triphenylene. Chem. Commun. 101-103 (2005).

21. Alam, M. A. et al. "Bicontinuous cubic" liquid crystalline materials from discotic molecules: a special effect of paraffinic side chains with ionic liquid pendants. J. Am. Chem. Soc. 131, 17722-17723 (2009).

22. Hatsusaka, K., Ohta, K., Yamamoto, I. \& Shirai, H. Discotic liquid crystals of transition metal complexes, part 30: spontaneous uniform homeotropic alignment of octakis(dialkoxyphenoxy)phthalocyaninatocopper(II) complexes. J. Mater. Chem. 11, 423-433 (2001)

23. Hatsusaka, K., Kimura, M. \& Ohta, K. Discotic liquid crystals of transition metal complexes 33: spontaneous uniform homeotropic alignment and unique mesophase transition behaviour of bis[2,3,9,10,16,17,23,24-octakis $(3,4$ dialkoxyphenoxy)-phthalocyaninato] lutetium(III) complexes. Bull. Chem. Soc. Jpn. 76, 781-787 (2003).

24. Ichihara, M., Suzuki, A., Hatsusaka, K. \& Ohta, K. Discotic liquid crystals of transition metal complexes 37: a thermotropic cubic mesophase having $P n \overline{3} m$ symmetry exhibited by phthalocyanine-based derivatives. Liq. Cryst. 34, 555-567 (2007)

25. Kato, T. \& Kamikawa, Y. Handbook of Liquid Crystals 2nd edn, Vol. 5, Ch. 10 (Wiley-VCH, Weinheim 2014).

26. Mansueto, M. \& Laschat, S. Handbook of Liquid Crystals 2nd edn, Vol. 6, Ch. 8 (Wiley-VCH, Weinheim 2014). 
27. Goossens, K., Lava, K., Bielawski, C. W. \& Binnemans, K. Ionic liquid crystals: versatile materials. Chem. Rev. 116, 4643-4807 (2016).

28. Kato, T., Uchida, J., Ichikawa, T. \& Sakamoto, T. Functional liquid crystals towards the next generation of materials. Angew. Chem. Int. Ed. 57, 4355-4371 (2018).

29. Cristadoro, A., Lieser, G., Joachim, H. \& Müllen, K. Field-force alignment of disc-type $\pi$ systems. ChemPhysChem 8, 586-591 (2007).

30. Heiney, P. A. Handbook of Liquid Crystals 2nd edn, Vol. 4, Ch. 10 (Wiley$\mathrm{VCH}$, Weinheim 2014).

31. Hayashi, H. Chemical reactions and magnetic fields. IEEE Transl. J. Magn. Jpn. 7, 586-590 (1992)

32. Kato, K. \& Tanaka, H. Visualizing charge densities and electrostatic potentials in materials by synchrotron X-ray powder diffraction. Adv. Phys. X 1, 55-80 (2016).

33. Miura, H. CellCalc: a unit cell parameter refinement program on Windows computer. J. Crystallogr. Soc. Jpn. 45, 145-147 (2003).

34. Yabashi, M. et al. Design of a beamline for the SPring-8 long undulator source 1. Nucl. Instrum. Methods Phys. Res. A 467-468, 678-681 (2001).

\section{Acknowledgements}

This work was supported by a Grant-in-Aid for Scientific Research on Innovative Areas " $\pi$-Figuration" (26102008) from The Ministry of Education, Culture, Sports, Science and Technology (MEXT), Japan and KAKENHI (24655118, 25247054 and 17H01034) from the Japan Society for the Promotion of Science (JSPS). This work was also supported in part by "Dynamic Alliance for Open Innovation Bridging Human, Environment and Materials" from MEXT, Japan. T.F. thanks the Tokuyama Science Foundation and the Sumitomo Foundation for financial support. T. Aida is grateful for a JSPS Grant-in-Aid for Specially Promoted Research on the Physically Perturbed Assembly for Tailoring High-Performance Soft Materials with Controlled Macroscopic Structural Anisotropy (25000005). F.H. thanks the World Premier International Research Center (WPI) Initiative on Materials Nanoarchitectonics and the International Center for Young Scientists (ICYS). The synchrotron XRD experiments were performed at the BL19LXU, BL44B2 and BL45XU beamlines at SPring- 8 with the approval of the RIKEN SPring- 8 Center (proposal numbers 20120045, 20130025, 20140056, 20150068, 20160027 and 20170055) and the Japan Synchrotron Radiation Research Institute (JASRI; proposal number 2014B1255). We thank Prof. K. Kadowaki and Dr. T. Kashiwagi (The University of Tsukuba) for FIR measurements. We are grateful to Prof. G. Ungar and Dr. X. Zeng (The University of Sheffield) for valuable discussion about structural characterization.

\section{Author contributions}

T.F. and T. Aida conceived the project. F.H., T.K. and T.F. designed the molecules and experiments. T.K., F.H., H.O., Y.T., K.K. and M.T. contributed to the X-ray diffraction measurements at SPring-8, and T.K. analysed the data. H.K., F.H. and T.K. contributed to the SQUID measurements, and T.K. and T. Arima analysed the data. F.H., T.K.,

T. Aida and T.F. co-wrote the manuscript.

\section{Additional information}

Supplementary Information accompanies this paper at https://doi.org/10.1038/s41467018-06976-7.

Competing interests: The authors declare no competing interests.

Reprints and permission information is available online at http://npg.nature.com/ reprintsandpermissions/

Publisher's note: Springer Nature remains neutral with regard to jurisdictional claims in published maps and institutional affiliations.

(c) (i) Open Access This article is licensed under a Creative Commons Attribution 4.0 International License, which permits use, sharing, adaptation, distribution and reproduction in any medium or format, as long as you give appropriate credit to the original author(s) and the source, provide a link to the Creative Commons license, and indicate if changes were made. The images or other third party material in this article are included in the article's Creative Commons license, unless indicated otherwise in a credit line to the material. If material is not included in the article's Creative Commons license and your intended use is not permitted by statutory regulation or exceeds the permitted use, you will need to obtain permission directly from the copyright holder. To view a copy of this license, visit http://creativecommons.org/ licenses/by/4.0/.

(C) The Author(s) 2018 